\title{
A Numerical-Experimental Method for Drop Impact Analysis of Composite Landing Gear
}

\author{
Yongliang Guan, ${ }^{1,2}$ Zhipeng Xue, ${ }^{1,3}$ Ming Li, ${ }^{1,3}$ and Hongguang Jia ${ }^{1,3}$ \\ ${ }^{1}$ Changchun Institute of Optics, Fine Mechanics and Physics, Chinese Academy of Sciences, 3888 Dong Nanhu Road, \\ Changchun 130033, China \\ ${ }^{2}$ University of Chinese Academy of Sciences, 19 A Yuquan Road, Beijing 100049, China \\ ${ }^{3}$ Chang Guang Satellite Technology Co., Ltd., Bei Yuanda Street and Longhu Road Interchange, Changchun 130052, China
}

Correspondence should be addressed to Hongguang Jia; 395227649@qq.com

Received 4 January 2017; Revised 17 February 2017; Accepted 15 May 2017; Published 13 June 2017

Academic Editor: M. I. Herreros

Copyright (C) 2017 Yongliang Guan et al. This is an open access article distributed under the Creative Commons Attribution License, which permits unrestricted use, distribution, and reproduction in any medium, provided the original work is properly cited.

\begin{abstract}
The special material performance, manufacturing process, machining behavior, and operating condition of composite materials cause uncertainties to inevitably appear in the mechanical properties of composite structures. Therefore, variability in mechanical properties must be considered in a mechanical response analysis of composite structures. A method is proposed in this paper to predict the dynamic performance of composite landing gear with uncertainties using experimental modal analysis data and nonlinear static test data. In this method, the nonlinear dynamic model of the composite landing gear is divided into two parts, the linear and the nonlinear parts. An experimental modal analysis is employed to predict the linear parameters with a frequency response function, and the nonlinear parameters caused by large deflection are identified by a nonlinear static test with the nonlinear least squares method. To check the accuracy and practicability of the method, it is applied to drop impact simulations and tests of composite landing gear. The results of the simulations are in good agreement with the test results, which shows that the proposed method is perfectly suited for the dynamic analysis of composite landing gear.
\end{abstract}

\section{Introduction}

Landing gear is the undercarriage of an aircraft or spacecraft and may be used for either takeoff or landing. For aircraft, the landing gear supports the craft when it is not flying, allowing it to take off, land, and taxi without damage. The impact loads are extremely high when the aircraft is landing [1-4], and the aircraft equipment would be damaged if the highimpact loads were transmitted to the airframe. Therefore, the landing gear is designed to absorb and dissipate the kinetic energy of the landing impact, reducing the impact loads transmitted to the airframe. The need to design landing gear takes into account various requirements of strength, stability, and stiffness, so accurate analyses are needed in the design of landing gear.

Composite materials are widely used in aerospace, aircraft, marine, civil, automotive, sport [5], and even optical engineering [6-10]. This is due to their outstanding physical, mechanical, and thermal properties, particularly their high stiffness and strength to weight ratios, good fatigue strength, excellent corrosion resistance, and dimensional stability. Composite materials frequently appear in impact condition. Increasing numbers of landing gears are being made of composite materials in the aviation field.

The dynamic characteristic analysis of the composite structures has recently attracted more attention. The finite element method is generally used for the analysis of complex structural behaviors of the composite structures. Many excellent theories are available on the finite element method for composite structural analysis. The classical analysis theory is based on the Kirchhoff plate theory, which is the simplest theory among them. The first-order shear deformation theory is suitable for the global structural behavior of thin and moderately thick laminated composite. Various higher-order shear deformation theories have overcome limitations in both the classical and first-order shear deformation theories. Layerwise lamination theory, which can predict the interlaminar stresses accurately, assumes a displacement representation 
formula in each layer, and the theory based on the 3D continuum can predict a composite laminate's interlaminar stress [11].

The theories have made significant progress but are based on accurate composite material parameters. The material properties determined from standard specimens tested in the laboratory may deviate significantly from those of actual laminated composite components manufactured in a factory [12]. Composite structures exhibit variability and uncertainties in their material properties with relation to their compositions and manufacturing processes [13]. Operating conditions, quality control procedures, and environmental effects are often difficult to control, which affect the performance of composite structures [14]. Delamination is a major problem in composite manufacturing which brings uncertainties in the material properties of composites [15]. The nonhomogeneous and anisotropic nature of composite materials make their machining behavior differ from metal machining [16], which can affect the mechanical properties of the composite structures [17]. Adhesive bonding is common practice in the interaction of composite parts, and its uncontrollable feature of consumption makes the parts' preformation discrete [18]. To ensure high reliability of the structures, the actual behaviors of the composite parts in service must be accurately predicted and carefully monitored. Therefore, it is essential to capture and model the variability and uncertainties inherent in these material properties.

The properties of composites can be obtained accurately via experimental tests [19], so the mixed numericalexperimental technique is applied to extract the physical information of composites. This usually involves minimizing an error function between the experimental and numerical outputs [13]. Constructing of predictive computational models for analysis and design of many complex engineering systems requires not only a fine representation of the relevant physics and their interactions but also a quantitative assessment of underlying uncertainties and their impact on design performance objectives [20]. Hence, a thorough characterization of the mechanical properties of these structures is needed to establish reliable designs. Soares et al. identified elastic properties of laminated composites by using experimental eigenfrequencies. The stiffness parameters were identified from the measured natural frequencies of the laminated composites plate by direct minimization of the identification function [21]. A similar method was used by Frederiksen and Araújo. Frederiksen improved the model used for identification [22]. Araújo used a mixed numericalexperimental technique to identify the damping properties of plyometric composites [23]. Cunha et al. identified several properties of a composite plate from a single test [24]. Diveyev et al. predicted elastic and damping properties of composite laminated plates on the basis of static three-point bending tests, measured eigenfrequencies, and refined calculation schemes [25]. Rikards et al. identified the elastic properties of cross-ply laminates from the measure eigenfrequencies of composite plates [26].

The mixed numerical-experimental technique makes the finite element method suitable for dynamic analysis of the laminated composites. However, the tests for measuring eigenfrequencies of composite plates are almost all linear, as in the works mentioned above, and do not consider the structural nonlinearities of the laminated composites. There are obvious geometrical nonlinearities in the work process of landing gears, so the nonlinear system identification is needed in the nonlinear structural models of landing gears. The nonlinear system identification in structural dynamics has been studied since the 1970s; many excellent methods are available for identification of nonlinear structural models. Time-domain methods, such as the restoring force surface method, the approach based on nonlinear autoregressive moving average with exogenous input model, and Hilbert transform-based data decomposition [27], have the advantage that the signals are directly provided by current measurement devices; less time and effort is spent on data acquisition and processing [28]. The frequency-domain methods consider the data from Fourier spectra, frequency response, and transmissibility functions, or power spectral densities, and extend modal analysis to nonlinear structures [28]. Timefrequency analysis offers useful insight into the dynamics of nonlinear systems [27]. Modal methods develop nonlinear system identification techniques based on nonlinear modes [28]. Black-box modeling concentrates on function which maps the input to the output [28]. Model updating methods improve the nonlinear dynamic modeling with test results [27].

Our study is aimed at expanding a numerical-experimental method based on linear and nonlinear tests for nonlinear modeling and drop impact analysis of composite landing gear.

\section{Numerical-Experimental Method}

The equation of motion of an $n$-degree-of-freedom nonlinear system can be written in the following form:

$$
\mathbf{M} \ddot{\mathbf{x}}(t)+\mathbf{C} \dot{\mathbf{x}}(t)+\left(\mathbf{K}+\mathbf{K}_{\mathbf{u}}\right) \mathbf{x}(t)=\mathbf{F}(t),
$$

where $\mathbf{M}$ and $\mathbf{K}$ are the real symmetric mass and stiffness matrices, respectively, $\mathbf{C}$ is the damping of the system, $\mathbf{x}(t)$ and $\mathbf{F}(t)$ are the displacements and exciting load vectors, respectively, and $\mathbf{K}_{\mathbf{u}}$ is the geometrically nonlinear stiffness component dependent on displacements. For the relevant problems, the nonlinear stiffness force vector $\mathbf{K}_{\mathbf{u}} \mathbf{x}(t)$ represents a deviation from the linear stiffness force vector $\mathbf{K x}(t)$ and is more than adequately represented by second- and third-order terms in $\mathbf{x}(t)$. When displacements are small, the second- and third-order terms become negligible and the total stiffness-related force vector is reduced to the regular linear term $\mathbf{K x}(t)$.

Any solution to (1) requires knowledge of the system matrices. In the linear identification works mentioned above, $\mathbf{M}, \mathbf{K}$, and $\mathbf{C}$ are generally available. The geometrically nonlinear stiffness is related to $\mathbf{K}_{\mathbf{u}}$, which is typically not available within a linear identification work. Therefore, a mean of numerically evaluating $\mathbf{M}, \mathbf{K}, \mathbf{C}$, and $\mathbf{K}_{\mathbf{u}}$ was developed. 
A set of coupled modal equations with reduced degrees of freedom is first obtained by applying the modal coordinate transformation:

$$
\mathbf{x}(t)=\boldsymbol{\Phi q}(t),
$$

where $\Phi$ is eigenvector matrices of the model defined in (1) without $\mathbf{K}_{\mathbf{u}}$ and $\mathbf{q}(t)$ is the vector of the displacement modal coordinates for each time instance $t$.

Generally, a subset of $l$ eigenvectors are included in the solution such that $l \leq n$, and $n$ is the number of physical degrees of freedom.

Then, (1) can be expressed as

$$
\widetilde{\mathbf{M}} \ddot{\mathbf{q}}(t)+\widetilde{\mathbf{C}} \dot{\mathbf{q}}(t)+\widetilde{\mathbf{K}} \mathbf{q}(t)+\gamma\left(q_{1}, q_{2}, \ldots, q_{l}\right)=\widetilde{\mathbf{F}}(t),
$$

where

$$
\begin{aligned}
\widetilde{\mathbf{M}} & =\boldsymbol{\Phi}^{T} \mathbf{M} \boldsymbol{\Phi}=\mathbf{I} \\
\widetilde{\mathbf{C}} & =\boldsymbol{\Phi}^{T} \mathbf{C} \boldsymbol{\Phi}=2 \boldsymbol{\xi} \boldsymbol{\omega} \\
\widetilde{\mathbf{K}} & =\boldsymbol{\Phi}^{T} \mathbf{K} \boldsymbol{\Phi}=\boldsymbol{\omega}^{2} \\
\boldsymbol{\gamma} & =\boldsymbol{\Phi}^{T} \mathbf{K}_{\mathbf{u}} \boldsymbol{\Phi} \\
\widetilde{\mathbf{F}}(t) & =\boldsymbol{\Phi}^{T} \mathbf{F}(t) .
\end{aligned}
$$

$q_{1}, q_{2}, \ldots, q_{l}$ are the components of $\mathbf{q}(t) . \boldsymbol{\omega}$ is the eigenvalue matrices of the model defined in (1) and $\xi$ is the damping ratio of the model. Then, (3) can be written as

$$
\begin{aligned}
\ddot{\mathbf{q}}(t) & +2 \xi \boldsymbol{\omega} \dot{\mathbf{q}}(t)+\boldsymbol{\omega}^{2} \mathbf{q}(t)+\boldsymbol{\gamma}\left(q_{1}, q_{2}, \ldots, q_{l}\right) \\
= & \boldsymbol{\Phi}^{\mathrm{T}} \mathbf{F}(t) .
\end{aligned}
$$

Equation (5) can be divided into two parts: the linear part

$$
\ddot{\mathbf{q}}(t)+2 \boldsymbol{\xi} \omega \dot{\mathbf{q}}(t)+\boldsymbol{\omega}^{2} \mathbf{q}(t)=\boldsymbol{\Phi}^{\mathrm{T}} \mathbf{F}_{m}(t)
$$

and the nonlinear part

$$
\gamma\left(q_{1}, q_{2}, \ldots, q_{l}\right)=\boldsymbol{\Phi}^{\mathrm{T}} \mathbf{F}_{s}(t)-\boldsymbol{\omega}^{2} \mathbf{q}(t),
$$

where $\mathbf{F}_{m}(t)$ is linear identification load and $\mathbf{F}_{s}(t)$ is nonlinear identification load.

In the numerical-experimental method of this paper, the linear part was identified by experimental eigenvalue matrices, eigenvector matrices, and others from experimental modal analysis, and the nonlinear part was identified by a nonlinear static test. The flowchart of the numericalexperimental method is shown in Figure 1.

2.1. Linear Parameters Identification. The linear parameters were identified by experimental modal analysis. The experimental modal analysis is a method to describe a structure in terms of its natural characteristics which are the frequencies, damping, and mode shapes. By using signal-analysis techniques, one can easily measure vibration on operating structures and make a frequency analysis.

The frequency spectrum is the description of how vibration levels vary with frequencies which can then be checked against a specification. The frequency response function measurement removes forces spectrum from the data and describes the inherent structural response between defined points on the structure.

In the time domain, structural properties are given in terms of mass, stiffness, and damping. The dynamic equilibrium of an $n$-degree-of-freedom system is generally given by

$$
\mathbf{M} \ddot{\mathbf{x}}(t)+\mathbf{C} \dot{\mathbf{x}}(t)+\mathbf{K x}(t)=\mathbf{F}(t) .
$$

The corresponding dynamic equilibrium in frequency domain can be described as

$$
\left(\mathbf{K}+i \omega \mathbf{C}-\omega^{2} \mathbf{M}\right) \mathbf{X}(\omega)=\mathbf{F}(\omega),
$$

where $\omega$ is the circular frequency of exciting loads and $\mathbf{X}(\omega)$ and $\mathbf{F}(\omega)$ are the Fourier transforms of output and input.

$$
\begin{aligned}
& \mathbf{X}(\omega)=\int_{-\infty}^{+\infty} \mathbf{x}(t) e^{-i \omega t} d t \\
& \mathbf{F}(\omega)=\int_{-\infty}^{+\infty} \mathbf{F}(t) e^{-i \omega t} d t
\end{aligned}
$$
as

The frequency response vector $\mathbf{X}(\omega)$ could be expressed

$$
\mathbf{X}(\omega)=\left(\mathbf{K}+i \omega \mathbf{C}-\omega^{2} \mathbf{M}\right)^{-1} \mathbf{F}(\omega) .
$$

The matrix $\mathbf{H}(\omega)$ is usually called the frequency response matrix.

$$
\mathbf{H}(\omega)=\left(\mathbf{K}+i \omega \mathbf{C}-\omega^{2} \mathbf{M}\right)^{-1}
$$

and the frequency response function model is the ratio of output/input spectra. Then, $\boldsymbol{\omega}$ and $\boldsymbol{\Phi}$, which are the eigenvalue and eigenvector matrices of the model, could be obtained from $\mathbf{H}(\omega)$.

2.2. Nonlinear Parameters Identification. The nonlinear parameters were identified by a nonlinear static test. This test measures the nonlinear displacements of the composite structure $\mathbf{x}(t)$ under different loads $\mathbf{F}_{\mathbf{s}}(t)$.

The equation of an $n$-degree-of-freedom under static loads can be seen as in (7), shown above.

$$
\gamma\left(q_{1}, q_{2}, \ldots, q_{l}\right)=\Phi^{\mathbf{T}} \mathbf{F}_{s}(t)-\omega^{2} \mathbf{q}(t) .
$$

$\boldsymbol{\omega}$ and $\boldsymbol{\Phi}$, being the eigenvalue and eigenvector matrices of the dynamic system, are obtained from linear parameters identification. $\mathbf{F}_{\mathbf{s}}(t)$ and $\mathbf{x}(t)$ are obtained from the nonlinear static test, and $\mathbf{q}(t)$ is converted from

$$
\mathbf{x}(t)=\boldsymbol{\Phi} \mathbf{q}(t) .
$$

Muravyov [29] and Kuether et al. [30] wrote the dynamic equations in modal coordinates and determined nonlinear stiffness coefficients by combining the linear modes. For the dynamic analysis, the process of obtaining the nonlinear stiffness coefficients replaces the procedure of solving the change of modal shapes and frequencies caused by deflection, 


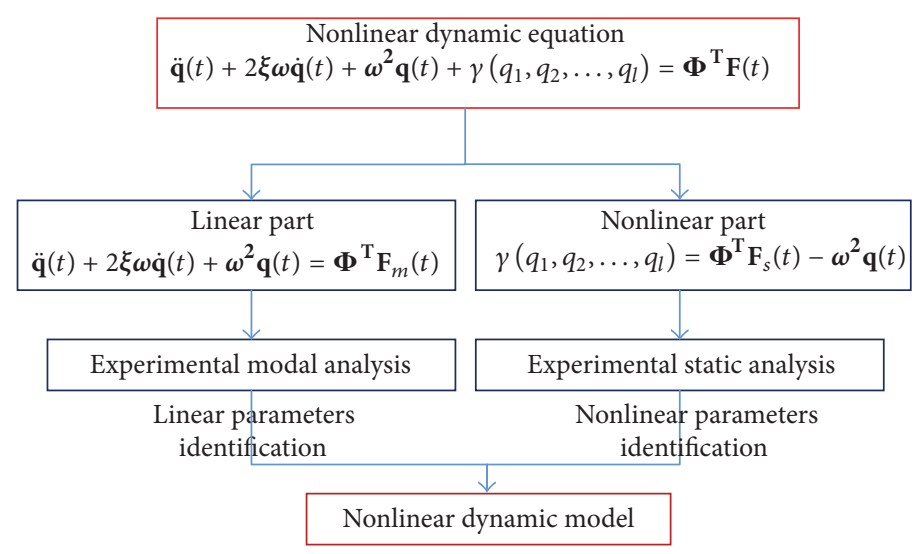

FIGURE 1: The flowchart of the numerical-experimental method.

and the verification results in their works show that the method has enough accuracy for the dynamic analysis.

The nonlinear force vector may be expressed in the following form:

$$
\begin{aligned}
\gamma\left(q_{1}, q_{2}, \ldots, q_{l}\right)= & \sum_{j=1}^{l} \sum_{k=j}^{l} a_{j k}^{r} q_{j} q_{k} \\
& +\sum_{j=1}^{l} \sum_{k=j}^{l} \sum_{m=k}^{l} b_{j k m}^{r} q_{j} q_{k} q_{m} \\
= & \boldsymbol{\Phi}^{\mathrm{T}} \mathbf{F}_{s}(t)-\boldsymbol{\omega}^{2} \mathbf{q}(t),
\end{aligned}
$$

where $a^{r}$ and $b^{r}$ are the $r$ th line identification parameters of $\gamma$, and $r=1,2, \ldots, l$. $l$ is the number of modes used in parameters identification.

The nonlinear parameters $a$ and $b$ in (15) were identified by the nonlinear static test based on the nonlinear least squares method. The nonlinear least squares method is one of the most common methods used in practical data analysis and involves the fitting of a theoretical model to experimental data. Frequently, the model takes the form of a dependent variable expressed as a function of several independent variables. Often the model will contain one or more estimated parameters. This estimation occurs on the basis of fitting the model to observations using the least squares concept.

There is a model of the following form:

$$
\mathbf{Q}=\mathbf{f}(K, L ; \alpha, \beta),
$$

where $\mathbf{Q}$ is viewed as a function of two independent variables $K$ and $L$ and $\alpha$ and $\beta$ are two constants. The objective is to estimate the values of the parameters $\alpha$ and $\beta$, which we shall do by minimizing the sum of squared errors $S$ over the allowable values of $\alpha$ and $\beta$ :

$$
S=S(\alpha, \beta)=\sum[f(K, L ; \alpha, \beta)-Q]^{2} .
$$

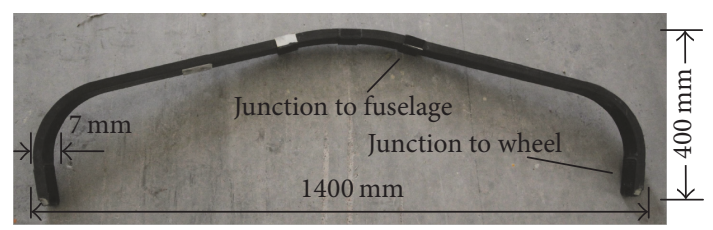

FIGURE 2: The composite landing gear.

The first-order necessary condition for a minimum is

$$
\begin{aligned}
& \frac{\partial S}{\partial \alpha}=0 \\
& \frac{\partial S}{\partial \beta}=0 .
\end{aligned}
$$

Then, the values of $K$ and $L$ are solved out.

The nonlinear dynamic model is built after the linear parameters $\boldsymbol{\omega}, \boldsymbol{\xi}$, and $\boldsymbol{\Phi}$ and nonlinear parameters $\mathbf{a}$ and $\mathbf{b}$ are identified.

\section{Identification of Composite Landing Gear}

3.1. Composite Landing Gear. The composite landing gear, as shown in Figure 2, is considered as follows. The length of the landing gear is $1400 \mathrm{~mm}$, the height is $400 \mathrm{~mm}$, and the thickness is $17 \mathrm{~mm}$. The top of the landing gear is fixed to the fuselage, and the bottom is the junction to wheels.

Three composite materials, the carbon cloth, the glass cloth, and the glass fiber with polymeric matrix, are used in the manufacture of the landing gear, as shown in Figure 3.

3.2. Linear Parameters Identification of the Landing Gear. The linear parameters of the composite landing gear were identified by experimental modal analysis. In general, the database is obtained from direct measurements. The frequency response functions are the measured output that will be used to construct the experimental realizations of the linear parameters identification.

The landing gear was tested in fixed constraint at the junction to the fuselage, as shown in Figure 4. 


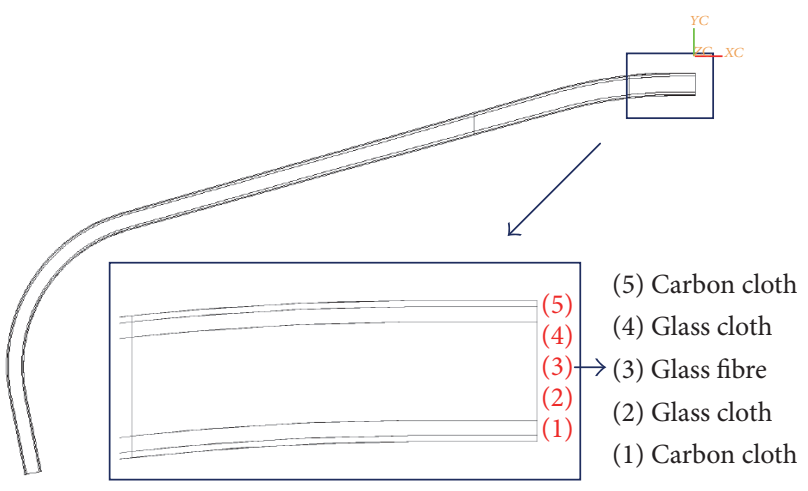

FIGURE 3: Composition of the landing gear.

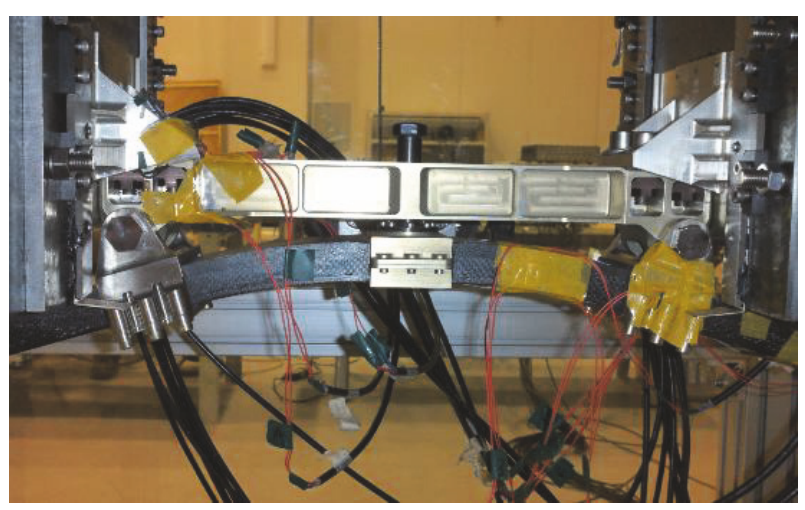

FIGURE 4: Setup of experimental modal analysis.

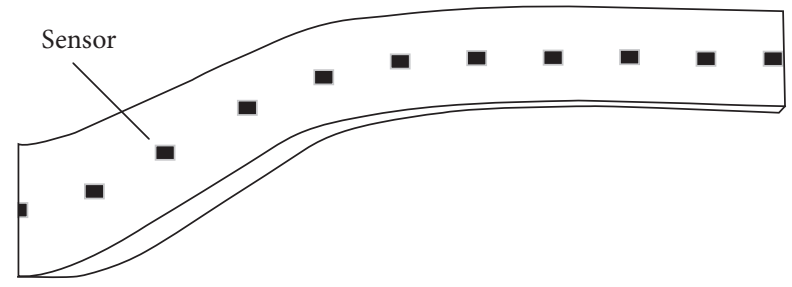

FIGURE 5: Locations of sensors.

The sensors were located in the center of both sides of the landing gear and separated by $20 \mathrm{~mm}$, as shown in Figure 5 .

The FRFs (frequency response functions) were computed by a data acquisition and analysis system LMS SCADAS III, and the experimental natural frequencies and modal shapes were obtained by modal analyses using LMS Test.Lab Structure analysis.

The first six modal shapes are shown in Figure 6.

Table 1 gives the first twenty frequencies of each mode.

3.3. Nonlinear Parameters Identification of the Landing Gear. The nonlinear parameters were identified by nonlinear static test. The test was performed in fixed constraint at the junction to the fuselage, and vertical downward loads were applied on the junction to the wheel, as shown in Figure 7.

The displacement of the landing gear changed as the load changed. The loads varied from $100 \mathrm{~N}$ to $1000 \mathrm{~N}$ with
TABLE 1: Frequency versus mode number.

\begin{tabular}{lc}
\hline Mode & Frequency/Hz \\
\hline 1 & 34.09 \\
2 & 78.52 \\
3 & 84.00 \\
4 & 137.81 \\
5 & 142.06 \\
6 & 214.93 \\
7 & 288.72 \\
8 & 356.38 \\
9 & 433.49 \\
10 & 435.64 \\
11 & 590.66 \\
12 & 658.78 \\
13 & 666.52 \\
14 & 769.27 \\
15 & 824.36 \\
16 & 961.63 \\
17 & 1068.71 \\
18 & 1165.20 \\
19 & 1172.93 \\
20 & 1303.38 \\
\hline
\end{tabular}

TABLE 2: Results of the nonlinear static test.

\begin{tabular}{lc}
\hline Load/N & Displacement $/ \mathrm{mm}$ \\
\hline 100 & 2.68 \\
200 & 5.36 \\
300 & 7.98 \\
400 & 10.76 \\
500 & 14.12 \\
600 & 17.28 \\
700 & 19.35 \\
800 & 23.55 \\
900 & 25.49 \\
1000 & 28.61 \\
\hline
\end{tabular}

increment of $100 \mathrm{~N}$. The displacements were measured by height caliper, and the displacements under different loads are shown in Table 2.

The nonlinear parameters of the composite landing gear were identified by (15) with the nonlinear least squares method based on the results of the nonlinear static test. Four modes were used to identify the nonlinear parameters of the composite landing gear.

$$
\begin{aligned}
\gamma\left(q_{1}, q_{2}, \ldots, q_{l}\right)= & \sum_{j=1}^{l} \sum_{k=j}^{l} a_{j k}^{r} q_{j} q_{k} \\
& +\sum_{j=1}^{l} \sum_{k=j}^{l} \sum_{m=k}^{l} b_{j k m}^{r} q_{j} q_{k} q_{m} \\
= & \boldsymbol{\Phi}^{\mathrm{T}} \mathbf{F}_{s}(t)-\boldsymbol{\omega}^{2} \mathbf{q}(t) .
\end{aligned}
$$



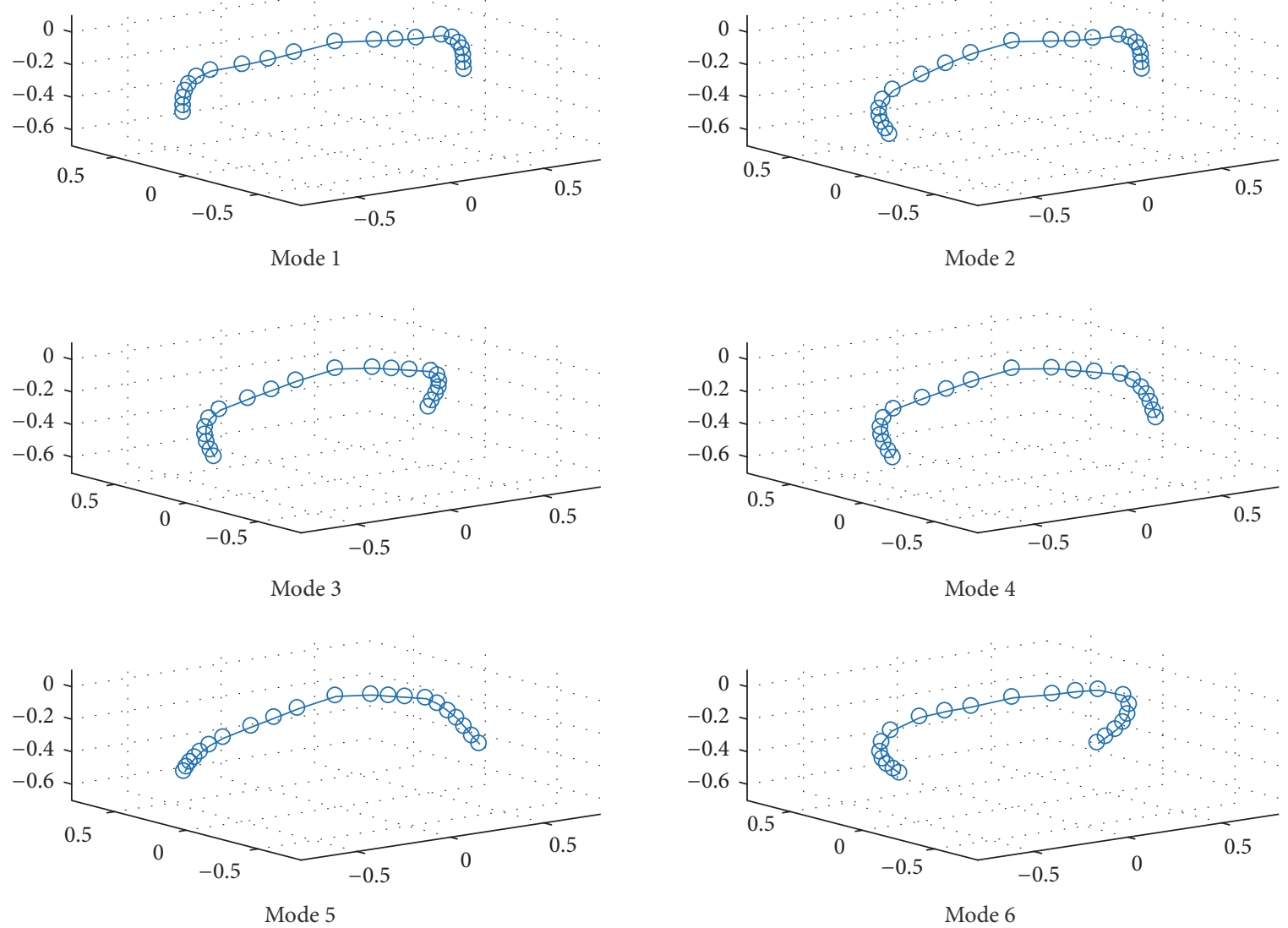

FIGURE 6: Modal shapes of the composite landing gear.

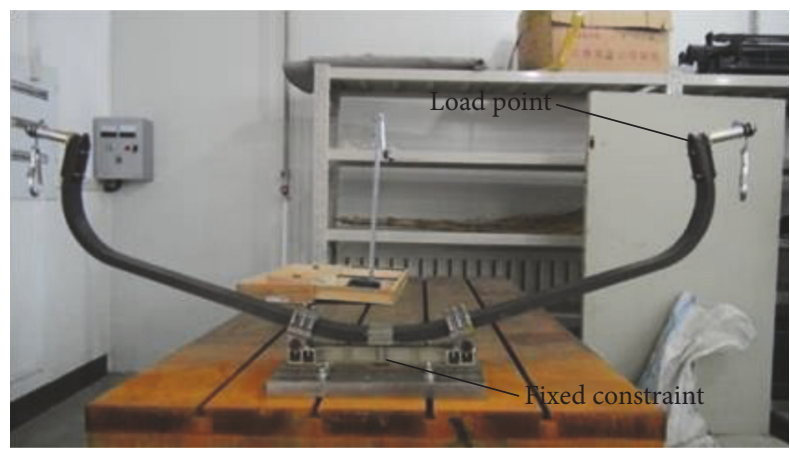

FIgURE 7: Setup of nonlinear static test.

The displacements of the landing gear under different loads were recalculated after the nonlinear parameters were identified. The results of the identification were compared with the displacement-loading curve of the nonlinear static test, as shown in Figure 8.

Figure 8 shows that the results of the identification are not obviously different from the results of the nonlinear static structural test.

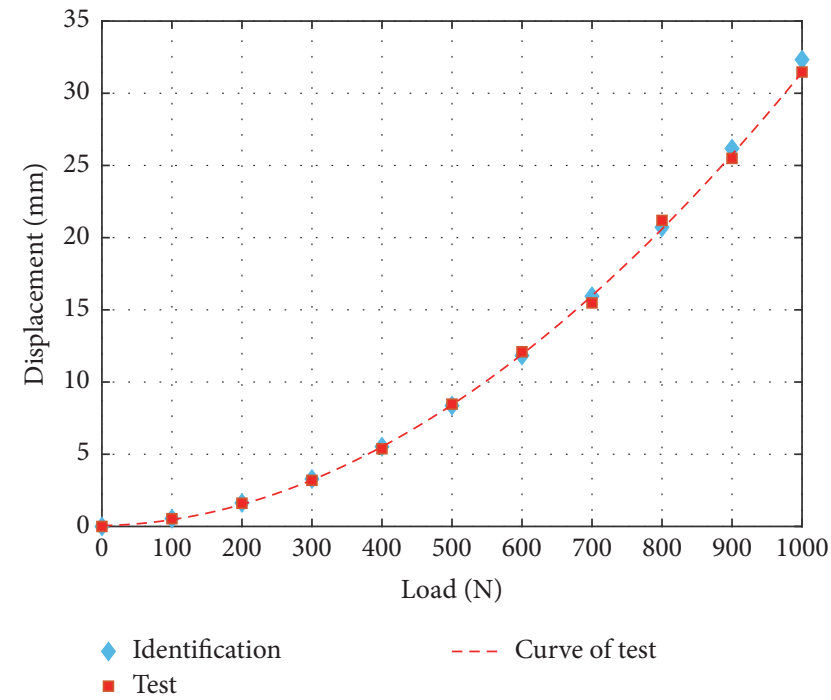

FIgURE 8: Results comparison of identification and test.

3.4. Tire Modeling. The tire modeling of the land gear is classical tire theory, which is based on the work of Mitschke and Wallentowitz [31]. In their work, a penetration formulation is 


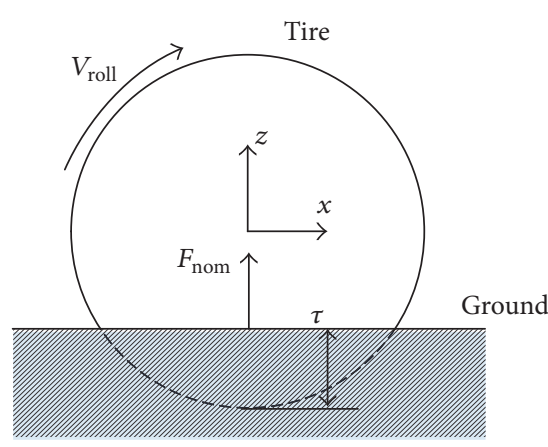

FIgURE 9: Tire modeling.

TABLE 3: Tire parameters.

\begin{tabular}{lc}
\hline Parameter & Value \\
\hline Radius & $0.1 \mathrm{~m}$ \\
Width & $0.075 \mathrm{~m}$ \\
Vertical stiffness & $75000 \mathrm{~N} / \mathrm{m}$ \\
Lateral stiffness & $166.55 \mathrm{~N} /{ }^{\circ}$ \\
Mass & $1.5 \mathrm{~kg}$ \\
$I_{x x} / I_{y y}$ & $3.45 \times 10^{-3} \mathrm{~kg} \cdot \mathrm{m}^{2}$ \\
$I_{z z}$ & $5.62 \times 10^{-3} \mathrm{~kg} \cdot \mathrm{m}^{2}$ \\
Damping & $175 \mathrm{~N} /(\mathrm{m} / \mathrm{s})$ \\
\hline
\end{tabular}

used to calculate the normal force of the tire applied by the ground, as shown in Figure 9.

The normal force applied to the tire is proportional to the penetration depth and the penetration rate, described as

$$
F_{\text {nom }}=K_{\text {nom }} \tau+C_{\text {nom }} \dot{\tau},
$$

where $K_{\text {nom }}$ and $C_{\text {nom }}$ are the vertical stiffness and damping of tire, respectively, and $\tau$ is the penetration depth.

The lateral force of the tire is described as a function of slip angle. The slip angle $\theta$ is the included angle of the tire's velocity and the tires' longitudinal axes, as shown in Figure 9. The lateral force is applied to account for the side loading on the tire and can be written as

$$
F_{\text {lat }}=C_{\text {lat }} \theta,
$$

where $C_{\text {lat }}$ is the lateral stiffness factor of the tire.

The parameters of the tire used in our work were measured by experimental method and are shown in Table 3 .

\section{Drop Impact Analysis of the Composite Landing Gear}

The landing gear is designed to absorb and dissipate the kinetic energy of the landing impact, reducing the impact loads transmitted to the airframe. Drop impact analysis is the method most widely used to check performance of the landing gear. Therefore, three drop impact simulations of the composite landing gear were carried out with the nonlinear dynamic model identified in Section 3. To check the accuracy of the method, three drop impact tests were also conducted.
4.1. Method for Drop Simulations. The drop impact simulations were carried out with the identified nonlinear dynamic model. In the simulations, the composite landing gear was dropped from a certain height and the whole process dynamic response of the landing gear was calculated.

To solve the nonlinear dynamic model, (5) was projected in the modal space. The modal space could reduce the differential equation to first order.

The nonlinear part of (7) was written as

$$
\begin{aligned}
\gamma\left(q_{1}, q_{2}, \ldots, q_{l}\right)= & \sum_{j=1}^{l} \sum_{k=j}^{l} a_{j k}^{r} q_{j} q_{k} \\
& +\sum_{j=1}^{l} \sum_{k=j}^{l} \sum_{m=k}^{l} b_{j k m}^{r} q_{j} q_{k} q_{m} \\
= & \chi\left(q_{1}, q_{2}, \ldots, q_{l}\right) \mathbf{q}(t) .
\end{aligned}
$$

Then, the modal space of (5) was

$$
\dot{\mathbf{z}}(t)=\mathbf{D} \mathbf{z}(t)+\mathbf{D}_{x} \mathbf{z}(t)+\mathbf{V F}(t),
$$

where

$$
\begin{aligned}
\mathbf{z}(t) & =\left\{\begin{array}{c}
\mathbf{q}(t) \\
\dot{\mathbf{q}}(t)
\end{array}\right\} \\
\mathbf{V} & =\left\{\begin{array}{c}
0 \\
\boldsymbol{\Phi}^{\mathbf{T}}
\end{array}\right\} \\
\mathbf{D} & =\left[\begin{array}{cc}
0 & I \\
-\boldsymbol{\omega}^{2} & -2 \boldsymbol{\xi} \boldsymbol{\omega}
\end{array}\right] \\
\mathbf{D}_{x} & =\left[\begin{array}{cc}
0 & 0 \\
-\chi\left(q_{1}, q_{2}, \ldots, q_{l}\right) & 0
\end{array}\right] .
\end{aligned}
$$

Cacciola and Muscolino put forward a new approach to convert the modal space to iterative equation in discrete time [32]. Divide the time axis in small intervals of equal length $\Delta t$ and let $t_{0}, t_{1}, \ldots, t_{k}, t_{k+1}, \ldots$ be the division time. Then, the numerical solution of (23) can be written as follows:

$$
\begin{aligned}
\mathbf{z}_{k+1}= & \boldsymbol{\Gamma}_{1}(\Delta t) \mathbf{D}_{\mathbf{x}}\left(\mathbf{z}_{k+1}\right) \mathbf{z}_{k+1} \\
& +\left(\boldsymbol{\Theta}_{0}(\Delta t)+\boldsymbol{\Gamma}_{0}(\Delta t) \mathbf{D}_{\mathbf{x}}\left(\mathbf{z}_{k}\right)\right) \mathbf{z}_{k} \\
& +\boldsymbol{\Gamma}_{0}(\Delta t) \mathbf{V F}_{k}+\boldsymbol{\Gamma}_{1}(\Delta t) \mathbf{V F}_{k+1},
\end{aligned}
$$

where

$$
\begin{aligned}
\mathbf{z}_{k} & =\mathbf{z}\left(t_{k}\right) \\
\mathbf{F}_{k} & =\mathbf{F}\left(t_{k}\right), \\
\mathbf{L}(\Delta t) & =\left[\Theta_{0}(\Delta t)-\mathbf{I}\right] \mathbf{D}^{-1} \\
\Gamma_{0}(\Delta t) & =\left[\Theta_{0}(\Delta t)-\frac{1}{\Delta t} \mathbf{L}(\Delta t)\right] \mathbf{D}^{-1} \\
\Gamma_{1}(\Delta t) & =\left[\frac{1}{\Delta t} \mathbf{L}(\Delta t)-\mathbf{I}\right] \mathbf{D}^{-1} .
\end{aligned}
$$


$\boldsymbol{\Theta}_{0}(t)$ is the so-called transition or fundamental matrix which is given as

$$
\Theta_{0}(t)=\left[\begin{array}{cc}
-\mathbf{g}(t) \boldsymbol{\omega}^{2} & \mathbf{h}(t) \\
-\mathbf{h}(t) \boldsymbol{\omega}^{2} & \dot{\mathbf{h}}(t)
\end{array}\right],
$$

where $\mathbf{g}(t), \mathbf{h}(t), \dot{\mathbf{g}}(t)$, and $\dot{\mathbf{h}}(t)$ are diagonal matrices whose $j$ th elements are given, respectively, as

$$
\begin{aligned}
& g_{j}(t)=-\frac{1}{\omega_{j}^{2}} e^{-\xi_{j} \omega_{j} t}\left[\cos \left(\omega_{D, j} t\right)+\frac{\xi_{j} \omega_{j}}{\omega_{D, j}} \sin \left(\omega_{D, j} t\right)\right] \\
& h_{j}(t)=\dot{g}_{j}(t)=\frac{1}{\omega_{D, j}} e^{-\xi_{j} \omega_{j} t} \sin \left(\omega_{D, j} t\right) \\
& \dot{h}_{j}(t)=e^{-\xi_{j} \omega_{j} t}\left[\cos \left(\omega_{D, j} t\right)-\frac{\xi_{j} \omega_{j}}{\omega_{D, j}} \sin \left(\omega_{D, j} t\right)\right],
\end{aligned}
$$

and

$$
\omega_{D, j}=\omega_{j} \sqrt{1-\xi_{j}^{2}}
$$

is the $j$ th damped natural frequency.

Then, (25) can be written as follows:

$$
\begin{aligned}
\mathbf{z}_{k+1}= & \left(I-\boldsymbol{\Gamma}_{1}(\Delta t) \mathbf{D}_{\mathbf{x}}\left(\mathbf{z}_{k+1}\right)\right)^{-1} \\
& \cdot\left(\Theta_{0}(\Delta t)+\boldsymbol{\Gamma}_{0}(\Delta t) \mathbf{D}_{\mathbf{x}}\left(\mathbf{z}_{k}\right)\right) \mathbf{z}_{k} \\
& +\left(I-\boldsymbol{\Gamma}_{1}(\Delta t) \mathbf{D}_{\mathbf{x}}\left(\mathbf{z}_{k+1}\right)\right)^{-1} \\
& \cdot\left(\boldsymbol{\Gamma}_{0}(\Delta t) \mathbf{V F}_{k}+\boldsymbol{\Gamma}_{1}(\Delta t) \mathbf{V F}_{k+1}\right) .
\end{aligned}
$$

The solution process of (30) was nonlinear, so an iterative method was needed. The iterative method used here was the Newton Raphson method.

In general, we will be searching for one or more solutions to the equation:

$$
\begin{gathered}
\mathbf{F}(\mathbf{x})=0 \\
\Downarrow \\
f_{1}\left(x_{1}, x_{2}, \ldots, x_{n}\right)=0 \\
f_{2}\left(x_{1}, x_{2}, \ldots, x_{n}\right)=0 \\
\vdots \\
f_{n}\left(x_{1}, x_{2}, \ldots, x_{n}\right)=0 .
\end{gathered}
$$

Consider the Taylor-series expansion of the function $\mathbf{F}(x)$ about a value $\mathbf{x}=\mathbf{x}^{j}$ :

$$
\begin{aligned}
\mathbf{F}(\mathbf{x})= & \mathbf{F}\left(\mathbf{x}^{j}\right)+\left(\frac{\partial \mathbf{F}(\mathbf{x})}{\partial \mathbf{x}}\right)_{\mathbf{x}^{j}}\left(\mathbf{x}-\mathbf{x}^{j}\right) \\
& +\frac{1}{2}\left(\frac{\partial^{2} \mathbf{F}(\mathbf{x})}{\partial \mathbf{x}^{2}}\right)_{\mathbf{x}^{j}}\left(\mathbf{x}-\mathbf{x}^{j}\right)^{2}+O\left(\mathbf{x}^{3}\right) \mathbf{x}^{j}=0 .
\end{aligned}
$$

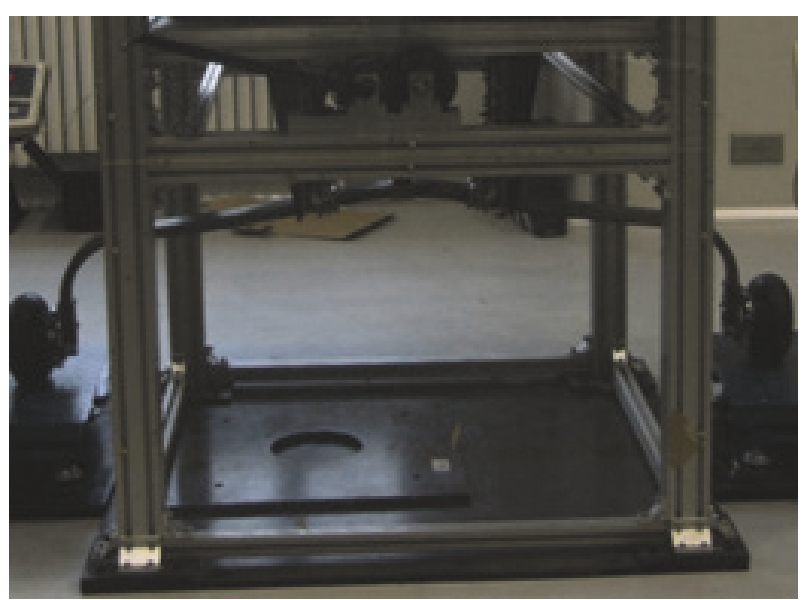

FIGURE 10: System of drop tests.

Using only the first two terms of the expansion, a first approximation to the root of (31) can be obtained from

$$
\mathbf{F}(\mathbf{x})=\mathbf{F}\left(\mathbf{x}^{j}\right)+\left(\frac{\partial \mathbf{F}(\mathbf{x})}{\partial \mathbf{x}}\right)_{\mathbf{x}^{j}}\left(\mathbf{x}-\mathbf{x}^{j}\right) \approx 0 .
$$

Such approximation is given by

$$
\mathbf{x} \approx \mathbf{x}^{j}-\left(\frac{\partial \mathbf{F}(\mathbf{x})}{\partial \mathbf{x}}\right)_{\mathbf{x}^{j}}^{-1} \mathbf{F}\left(\mathbf{x}^{j}\right) .
$$

The Jacobian matrix of $\mathbf{F}(\mathbf{x})$ is $\mathbf{J}(\mathbf{x})$ :

$$
\mathbf{J}(\mathbf{x})=\left[\begin{array}{cccc}
\frac{\partial f_{1}}{\partial x_{1}} & \frac{\partial f_{1}}{\partial x_{2}} & \cdots & \frac{\partial f_{1}}{\partial x_{n}} \\
\frac{\partial f_{2}}{\partial x_{1}} & \frac{\partial f_{2}}{\partial x_{2}} & \cdots & \frac{\partial f_{2}}{\partial x_{n}} \\
\vdots & \vdots & \vdots & \vdots \\
\frac{\partial f_{n}}{\partial x_{1}} & \frac{\partial f_{n}}{\partial x_{2}} & \cdots & \frac{\partial f_{n}}{\partial x_{n}}
\end{array}\right]
$$

Then, the solution of (31) is

$$
\mathbf{x}=\mathbf{x}-J(\mathbf{x})^{-1} \mathbf{F}(\mathbf{x}) .
$$

The convergence criterion of the iterative process was based on the minimum 2-norm. In the solution process of (30), while

$$
\left\|\mathbf{z}_{k+1}-\mathbf{z}_{k}\right\|_{2} \leq \text { CRIT }
$$

the results are convergent.

4.2. Setup of Drop Tests. The drop tests were carried out with the system shown in Figure 10, and the degrees of freedom except the vertical direction of the composite landing gear were limited by the drop-test platform.

The wheels were fixed to the bottom of the landing gear, and the equivalent mass of the aircraft was fixed to the junction to fuselage, as shown in Figure 11. 

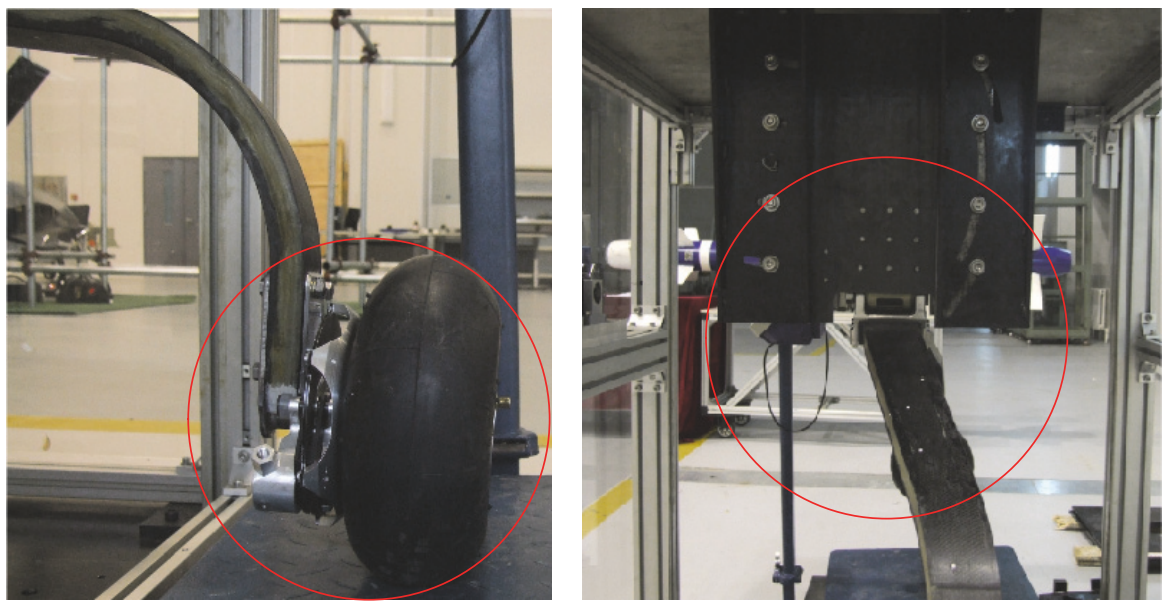

FIgURE 11: Setup of drop tests.

TABLE 4: Parameters of the drop impact analysis.

\begin{tabular}{lccc}
\hline Number & Equivalent mass $/ \mathrm{kg}$ & Height $/ \mathrm{m}$ & $\mathrm{AOA}^{\circ}$ \\
\hline $\mathrm{A}$ & 120 & 0.136 & 0 \\
$\mathrm{~B}$ & 131 & 0.46 & 0 \\
$\mathrm{C}$ & 136 & 0.46 & 12 \\
\hline
\end{tabular}

In drop tests, the composite landing gear was dropped from the same height as the simulations, and impact loads of the ground were applied to the wheels. The whole process dynamic response was collected by sensors, and the data collection system was LMS SCADAS III.

4.3. Drop Impact Analysis. To check the accuracy of the identified nonlinear dynamic model, three groups of drop impact analysis were carried out. Every group contained a drop simulation and a drop test. The drop simulations were based on the identified nonlinear dynamic model, and the experimental method mentioned above was used in the drop tests.

The analysis parameters of drop impact analysis are shown in Table 4.

Since the fuselage of the aircraft was replaced by the equivalent mass, the dynamic responses of the equivalent mass were measured in the drop impact analysis. In drop analysis $\mathrm{A}$, the dynamic responses of the equivalent mass after both the drop simulation and drop test were compared, and the results are shown in Figure 12.

Figure 12 shows that the result of the simulation is in good agreement with the result of the test, and the maximum error of the simulation compared to the test is $4.33 \%$.

The analysis parameters of the second drop impact analysis are different from those of the first analysis. The dynamic responses of the equivalent mass after both drop simulation and the drop test were compared; the results are shown in Figure 13.

Figure 13 shows that the result of the simulation is not obviously different from the result of the test, and the

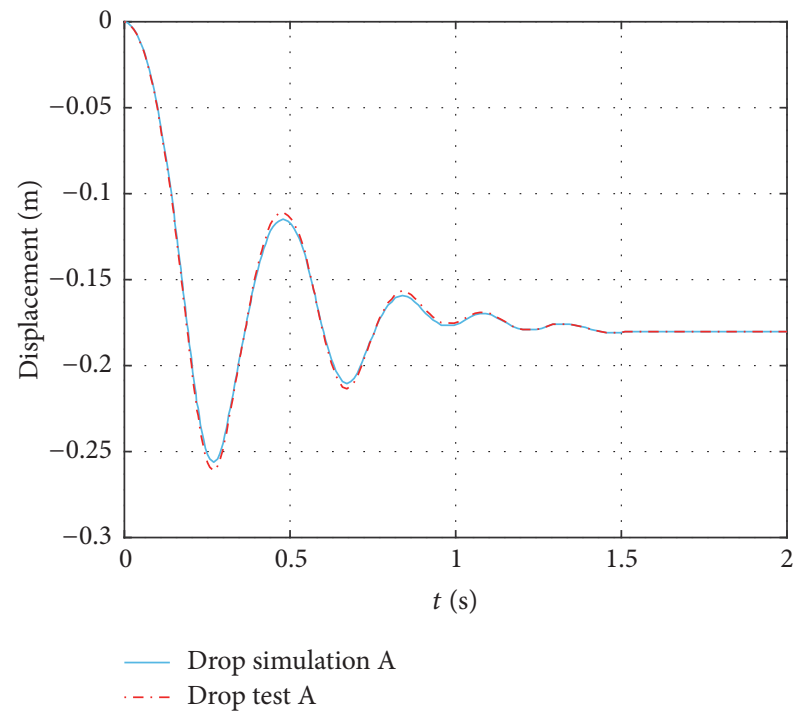

Figure 12: Comparison of results for drop analysis A.

maximum error of the simulation compared to the test is $5.36 \%$.

In the third drop impact analysis, the dynamic responses of the equivalent mass after both the drop simulation and drop test were compared, and the results are shown in Figure 14.

Figure 14 shows that the result of the simulation is in good agreement with the result of the test, and the maximum error of the simulation compared to the test is $4.55 \%$.

\section{Conclusion}

A method is proposed in this paper to predict the dynamic performance of composite landing gear with uncertainties using experimental modal analysis data and nonlinear static test data. In the method, the nonlinear dynamic model of the composite landing gear is divided into two parts: the linear and the nonlinear parts. Experimental modal analysis is 


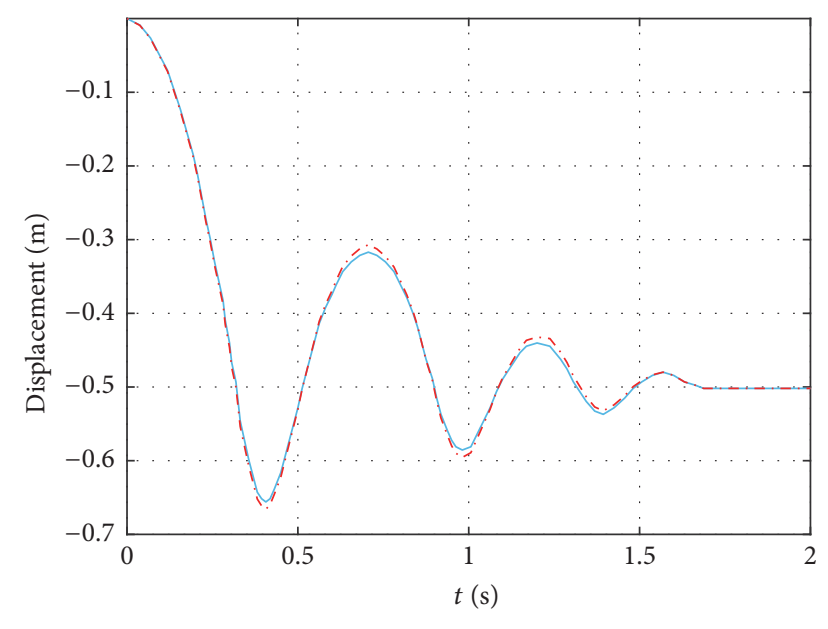

_ Drop simulation B

-.. Drop test B

FIgURE 13: Comparison of results for drop analysis B.

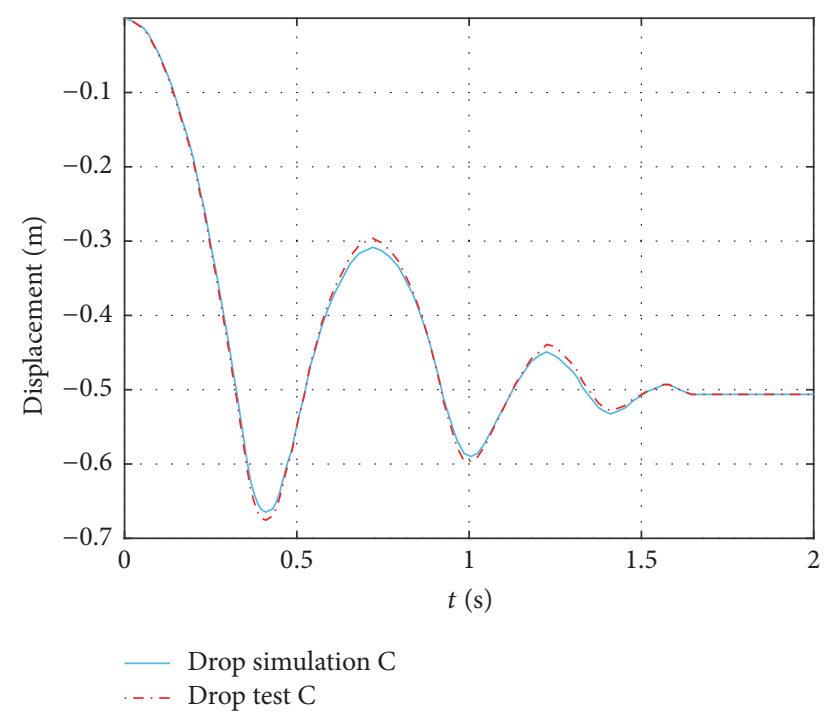

FIGURE 14: Comparison of results for drop analysis C.

employed to predict the linear parameters with a frequency response function and the geometrically nonlinear parameters are identified by nonlinear static test with the nonlinear least squares method.

To check its accuracy and practicability, the method is applied to drop impact analysis of composite landing gear. Both simulations and tests are conducted for the drop impact analysis, and the errors of the analyses are extremely small. The maximum error of the simulation compared to the test is $4.33 \%$ in drop analysis $\mathrm{A}$, the maximum error of drop analysis $\mathrm{B}$ is $5.36 \%$, and the maximum error of drop analysis $\mathrm{C}$ is $4.55 \%$. The results of the simulations are in good agreement with the test results, which shows that the proposed method can accurately model the dynamic performance of composite landing gear and the method is perfectly suitable for dynamic analysis of composite landing gear.

\section{Conflicts of Interest}

The authors declare that there are no conflicts of interest regarding the publication of this paper.

\section{Acknowledgments}

The project was supported by the Knowledge Innovation Program of the Chinese Academy of Sciences (Grant no. YYYJ-1122) and the Innovation Program of UAV funded by the Chang Guang Satellite Technology Co., Ltd. The authors also gratefully acknowledge the support from the Science Fund for Young Scholars of the National Natural Science Foundation of China (Grant no. 51305421).

\section{References}

[1] Z.-P. Xue, M. Li, Y.-H. Li, and H.-G. Jia, "A simplified flexible multibody dynamics for a main landing gear with flexible leaf spring," Shock and Vibration, vol. 2014, Article ID 595964, 10 pages, 2014.

[2] X. M. Dong and G. W. Xiong, "Vibration attenuation of magnetorheological landing gear system with human simulated intelligent control," Mathematical Problems in Engineering, vol. 2013, Article ID 242476, 13 pages, 2013.

[3] G. Mikułowski and Ł. Jankowski, "Adaptive landing gear: optimum control strategy and potential for improvement," Shock and Vibration, vol. 16, no. 2, pp. 175-194, 2009.

[4] F. A. C. Viana, V. Steffen Jr., M. A. X. Zanini, S. A. Magalhães, and L. C. S. Góes, "Identification of a non-linear landing gear model using nature-inspired optimization," Shock and Vibration, vol. 15, no. 3-4, pp. 257-272, 2008.

[5] M. Ansar, W. Xinwei, and Z. Chouwei, "Modeling strategies of 3D woven composites: a review," Composite Structures, vol. 93, no. 8, pp. 1947-1963, 2011.

[6] G. Z. Dai, H. X. Xiao, W. Wu et al., "Plasmon-driven reaction controlled by the number of graphene layers and localized surface plasmon distribution during optical excitation," LightScience \& Applications, vol. 4, 2015.

[7] P. Lutsyk, R. Arif, J. Hruby et al., "A sensing mechanism for the detection of carbon nanotubes using selective photoluminescent probes based on ionic complexes with organic dyes," LightScience \& Applications, vol. 5, 2016.

[8] C. X. Ma, Y. Dai, L. Yu et al., "Energy transfer in plasmonic photocatalytic composites," Light-Science \& Applications, vol. 5, 2016.

[9] S. Malobabic, M. Jupé, and D. Ristau, "Spatial separation effects in a guiding procedure in a modified ion-beam-sputtering process," Light-Science and Applications, vol. 5, article e16044, 2016.

[10] F. J. Rodríguez-Fortuno and A. V. Zayats, "Repulsion of polarised particles from anisotropic materials with a near-zero permittivity component," Light-Science and Applications, vol. 5, article e16022, 2016.

[11] Y. X. Zhang and C. H. Yang, "Recent developments in finite element analysis for laminated composite plates," Composite Structures, vol. 88, no. 1, pp. 147-157, 2009.

[12] W. T. Wang and T. Y. Kam, "Material characterization of laminated composite plates via static testing," Composite Structures, vol. 50, no. 4, pp. 347-352, 2000. 
[13] L. Mehrez, D. Moens, and D. Vandepitte, "Stochastic identification of composite material properties from limited experimental databases, part I: experimental database construction," Mechanical Systems and Signal Processing, vol. 27, no. 1, pp. 471483, 2012.

[14] S. Sriramula and M. K. Chryssanthopoulos, "Quantification of uncertainty modelling in stochastic analysis of FRP composites," Composites Part A: Applied Science and Manufacturing, vol. 40, no. 11, pp. 1673-1684, 2009.

[15] P. K. Rakesh, V. Sharma, I. Singh, and D. Kumar, "Delamination in fiber reinforced plastics: a finite element approach," Engineering, vol. 3, no. 05, pp. 549-554, 2011.

[16] C. C. Tsao and H. Hocheng, "Taguchi analysis of delamination associated with various drill bits in drilling of composite material," International Journal of Machine Tools and Manufacture, vol. 44, no. 10, pp. 1085-1090, 2004.

[17] A. T. Marques, L. M. Durão, A. G. Magalhães, J. F. Silva, and J. M. R. S. Tavares, "Delamination analysis of carbon fibre reinforced laminates: Evaluation of a special step drill," Composites Science and Technology, vol. 69, no. 14, pp. 2376-2382, 2009.

[18] S. Pantelakis and K. I. Tserpes, "Adhesive bonding of composite aircraft structures: Challenges and recent developments," Science China: Physics, Mechanics and Astronomy, vol. 57, no. 1, pp. 2-11, 2014.

[19] D. Jiang, Y. Li, Q. Fei, and S. Wu, "Prediction of uncertain elastic parameters of a braided composite," Composite Structures, vol. 126, pp. 123-131, 2015.

[20] L. Mehrez, A. Doostan, D. Moens, and D. Vandepitte, "Stochastic identification of composite material properties from limited experimental databases, part ii: uncertainty modelling," Mechanical Systems and Signal Processing, vol. 27, pp. 484-498, 2012.

[21] C. M. M. Soares, M. M. de Freitas, A. L. Araújo, and P. Pedersen, "Identification of material properties of composite plate specimens," Composite Structures, vol. 25, no. 1-4, pp. 277285, 1993.

[22] P. S. Frederiksen, "Application of an improved model for the identification of material parameters," Mechanics of Composite Materials and Structures, vol. 4, no. 4, pp. 297-316, 1997.

[23] A. L. Araújo, C. M. Mota Soares, and M. J. Moreira De Freitas, "Characterization of material parameters of composite plate specimens using optimization and experimental vibrational data," Composites Part B: Engineering, vol. 27, no. 2, pp. 185-191, 1996.

[24] J. Cunha, S. Cogan, and C. Berthod, "Application of genetic algorithms for the identification of elastic constants of composite materials from dynamic tests," International Journal for Numerical Methods in Engineering, vol. 45, no. 7, pp. 891-900, 1999.

[25] B. Diveyev, I. Butiter, and N. Shcherbina, "Identifying the elastic moduli of composite plates by using high-order theories," Mechanics of Composite Materials, vol. 44, no. 1, pp. 25-36, 2008.

[26] R. Rikards, A. Chate, and G. Gailis, "Identification of elastic properties of laminates based on experiment design," International Journal of Solids and Structures, vol. 38, no. 30, pp. 5097-5115, 2001.

[27] J. P. L. No and G. Kerschen, "Nonlinear system identification in structural dynamics: 10 more years of progress," Mechanical Systems \& Signal Processing, vol. 83, pp. 2-35, 2016.

[28] G. Kerschen, K. Worden, A. F. Vakakis, and J. Golinval, "Past, present and future of nonlinear system identification in structural dynamics," Mechanical Systems and Signal Processing, vol. 20, no. 3, pp. 505-592, 2006.

[29] A. A. Muravyov, "Determination of nonlinear stiffness coefficients for finite element models with application to the random vibration problem," MSC Worldwide Aerospace Conference, pp. $1-14,1999$.

[30] R. J. Kuether, B. J. Deaner, J. J. Hollkamp, and M. S. Allen, "Evaluation of geometrically nonlinear reduced-order models with nonlinear normal modes," AIAA Journal, vol. 52, no. 11, pp. 3273-3285, 2015.

[31] M. Mitschke and H. Wallentowitz, Dynamik der Kraftfahrzeuge, Springer, Berlin, Germany, 1972.

[32] P. Cacciola and G. Muscolino, "Dynamic response of a rectangular beam with a known non-propagating crack of certain or uncertain depth," Computers and Structures, vol. 80, no. 27, pp. 2387-2396, 2002. 


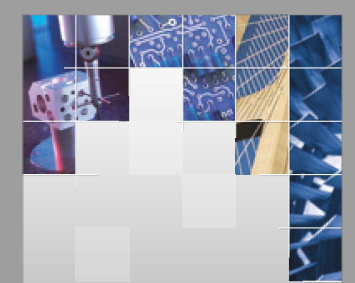

\section{Enfincering}
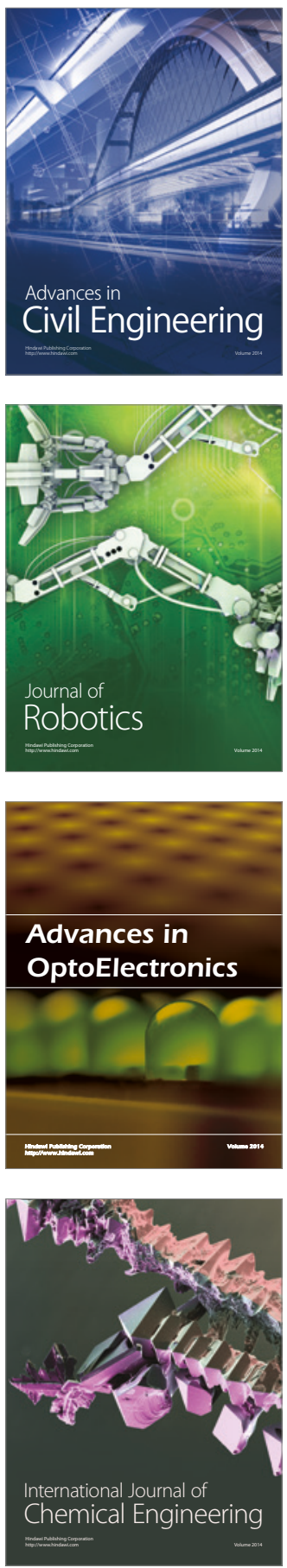

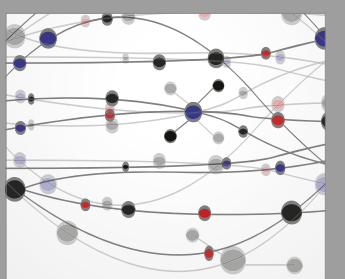

The Scientific World Journal

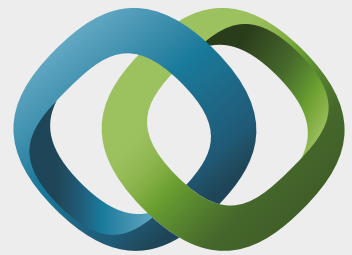

\section{Hindawi}

Submit your manuscripts at

https://www.hindawi.com
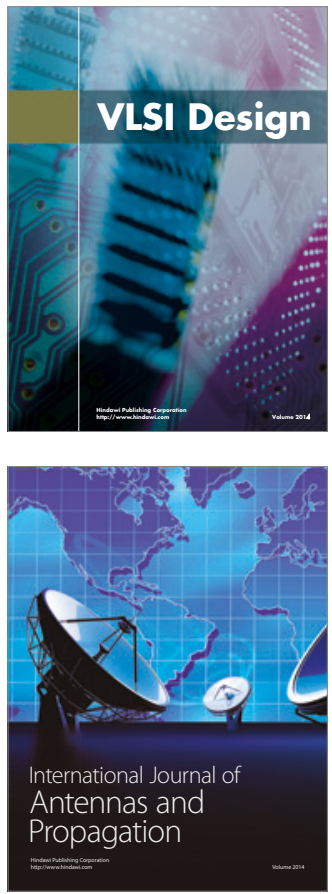

\section{Rotating}

Machinery
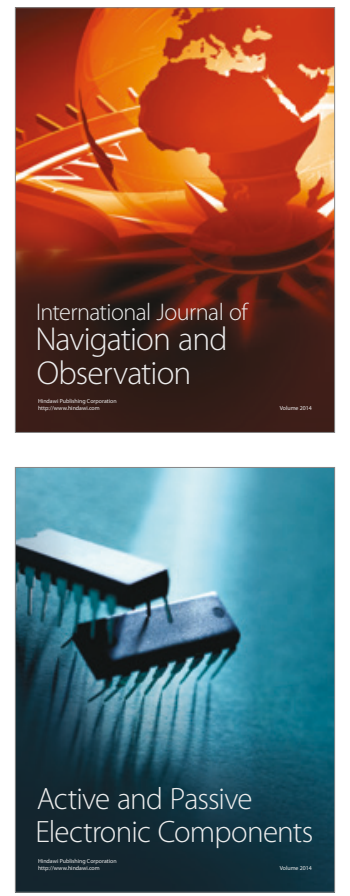
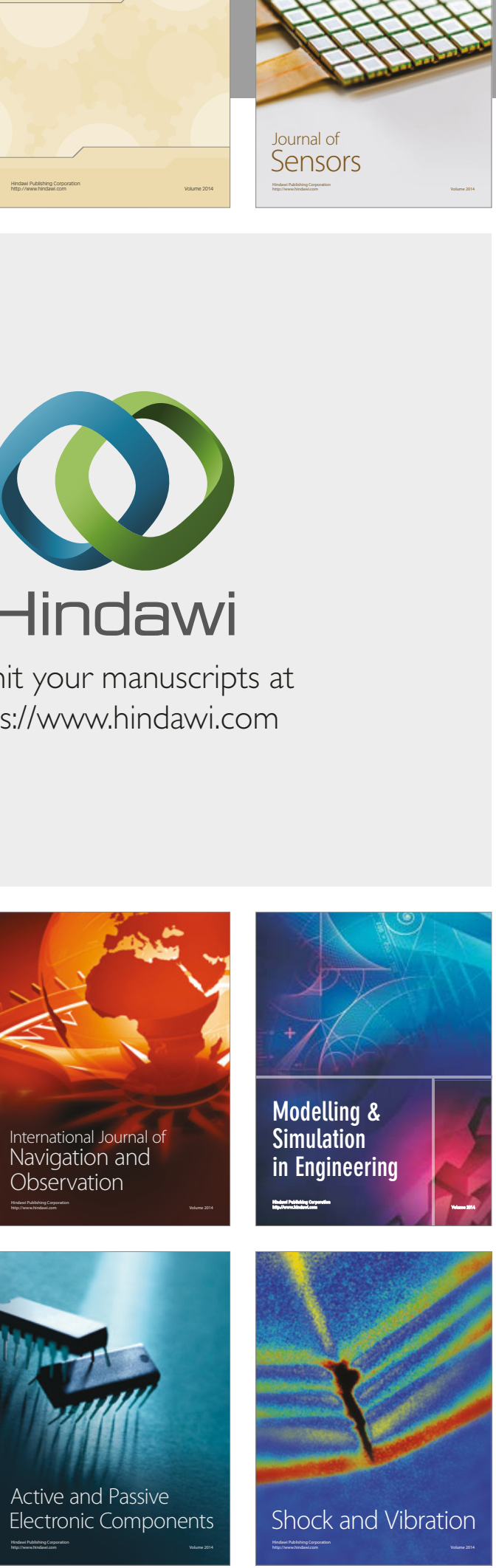
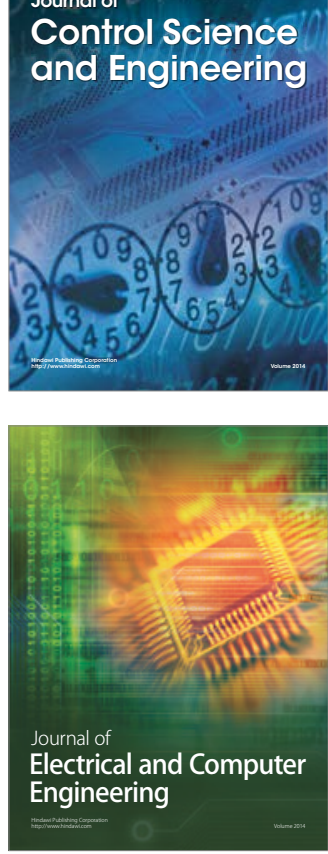

Distributed

Journal of

Control Science

and Engineering
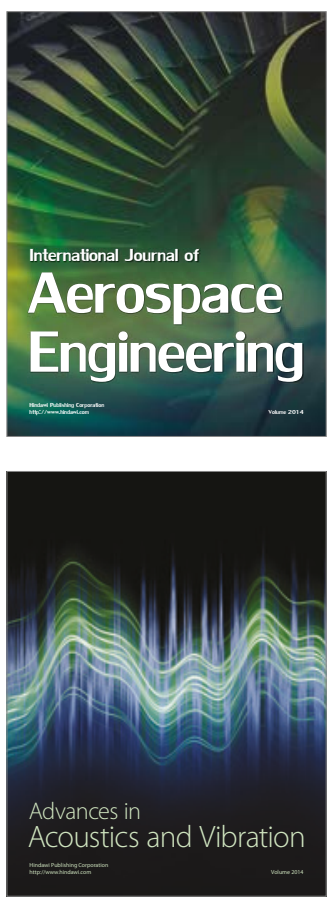

Sensor Networks 